\title{
Low Cost 3D Scanners Along the Design of Lower Limb Prosthesis
}

\author{
Claudio COMOTTI ${ }^{1}$, Giorgio COLOMBO ${ }^{2}$, Daniele REGAZZON।*1, Caterina RIZZI ${ }^{1}$, Andrea VITALI $^{1}$ \\ ${ }^{1}$ University of Bergamo, Dalmine (BG), Italy; ${ }^{2}$ Politecnico of Milano, Milan, Italy \\ DOI: 10.15221/15.147 http://dx.doi.org/10.15221/15.147
}

\begin{abstract}
The evolution of 3D scanning systems has determined a large range of commercial solutions available on the market with different costs depending on their performances. The most interesting scanners for the sake of this research rely on structured light optical sensors like Microsoft Kinect v1 sensor, which are extremely low-cost, but they still provide a precision that is valuable for some medical applications, e.g., the scanning of a residual limb.

In this research work, we present our CAD system based on a knowledge-guided approach to design the socket starting from two different 3D acquisition systems; the first one uses Microsoft Kinect and the second one exploits MRI volumes to get the final 3D shape of residual limb. Then, a comparison of introduced techniques to create 3D shape is exposed. Final outcomes are shown and discussed in the paper.
\end{abstract}

Keywords: 3D scanning, socket, MRI, Kinect, residual limb model, lower limb prosthesis

\section{Introduction}

The growing diffusion of 3D scanning technologies represents an interesting opportunity in several application fields. We pay attention to orthopedic products that are strictly driven by human body shapes, such as for a lower limb prosthesis. Prosthesis design is composed by different steps among which the design of the socket is crucial. The socket is the most customized part because its shape must be defined according to the shape of the residual limb as well as other parameters relative to the evaluation of residual and anthropometric patient's data.

In the last decade, our research group has developed a software platform, named Socket Modelling Assistant (SMA), in order to emulate traditional manufacturing process for lower limb prosthesis. SMA makes available a set of virtual tools, which emulates operations made by orthopedic technicians during traditional workflow in an orthopedic laboratory. SMA starts prosthesis design from 3D model of residual limb, which can be obtained using either 3D portable scanner or MRI images.

The whole project follows the low cost philosophy and thus, the choice of 3D scanning systems has been centered on the use of cheap 3D systems on which we can develop our custom software modules for SMA. There are different solutions that have been tested into the system, such as Microsoft Kinect v1. This type of devices can use available a wide set of low-cost or free software applications that permit to reconstruct 3D models starting from the acquired point cloud of an object.

Our aim is to reach the best quality of the 3D virtual residual limb according to precision of measurement and the structure of mesh through the use of low cost IT systems and custom software modules developed within this research activity.

In this paper, firstly, we introduce the differences between traditional process manufacturing and our system SMA to design the socket of lower limb prosthesis. Then, a presentation of available 3D scanner will be introduced and discussed. The use of MRI volume and 3D scanner acquisition is discussed exposing a case study that takes in consideration a transfemoral (AK) patient. Finally, preliminary test and reached results will be discussed.

\section{Prosthesis development process}

\subsection{Traditional socket development process}

The prosthetic socket is the most critical component for both transfemoral and transtibial prostheses. It is manually made from a positive chalk mold of the residual limb. The plaster mold traditionally is realized using a fully handmade procedure.

In this process, the prosthetist optimizes the socket shape (Figure 1) to reach a full comfortable contact with the prosthesis by the patient.

The first phase consists in making some measurements of the residual limb circumferences and then it is possible to proceed with the manufacturing. In this step the technician creates a negative cast manipulating by hands plaster patches directly on patient's residual limb. After that, s/he makes manually the positive model. This plaster model is modified by adding and removing material in particular zones, according to residual limb measurements and patient's characteristics (e.g., muscle and soft areas). 
The next step is to obtain a check socket with thermo-formable material posed directly on the modified positive model and tested on the amputee patient. If any other modifications are required, those are made directly on the positive mold and generally, those are about comfort and fitting. Finally, the definitive socket is realized and all the prosthesis components are assembled for the final static set-up.
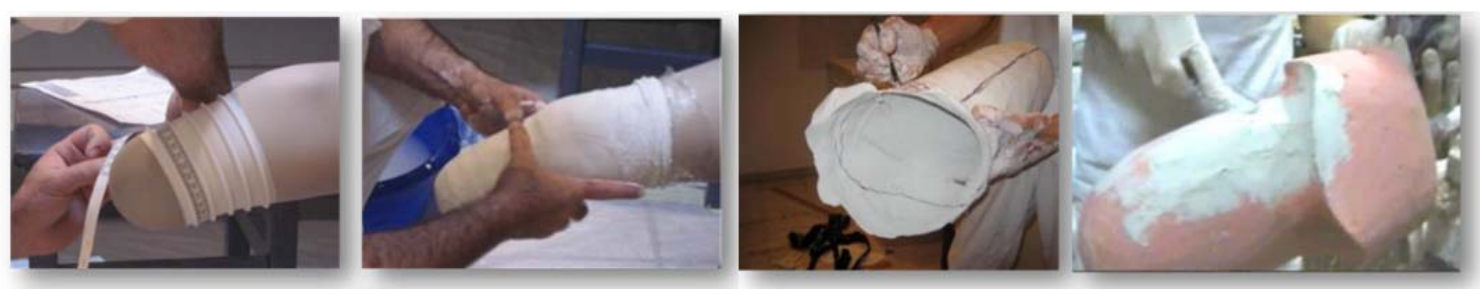

Figure 1 - Traditional socket development process

At present, there are several commercial CAD systems used for socket design that support some steps of traditional manufacturing process. They exploit expensive 3D laser scanner in order to acquire the 3D model of residual limb and thus, this model is used into CAD software to define the initial virtual positive 3D model of socket. The high cost of these solutions discourages small orthopedic labs that have not many funds to invest in these technologies. Furthermore, the use of these systems requires ad-hoc skills in CAD design, which do not belong by orthopedic technician. In fact, they design socket using knowledge that has been acquired with many years of experience.

In conclusion, the traditional socket development process is a very long procedure of about some weeks that is based on personal knowledge of prosthetist. Some CAD systems are available, but they are expensive and very difficult to use for orthopedic technician. Another important aspect is that the process is fully manual and during years it had not a significant evolution. The importance to reproduce the residual limb geometry is the main problem of the process.

\subsection{SMA: Socket Modeling Assistant}

In the last decade, our research group has been developing an ad-hoc knowledge-guided CAD system which allows the prosthetist to design the socket of both transtibial and transfemoral prosthesis relying on the digital model of the residual limb. Socket Modelling Assistant (SMA) is a virtual laboratory that emulates all phases of the socket development process like illustrated in Figure 2.

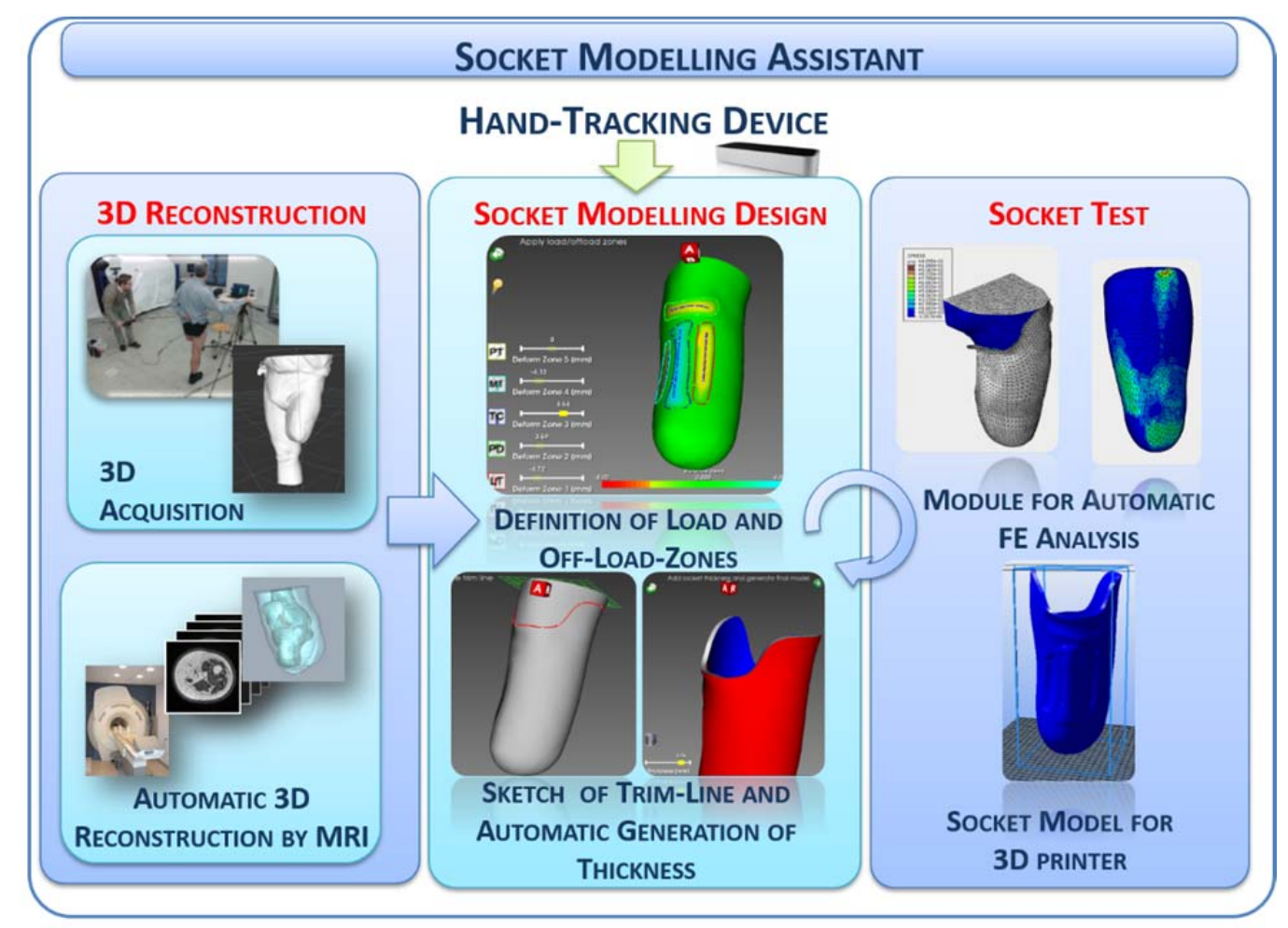

Figure 2 - SMA (Socket Modelling Assistant) 


\subsubsection{D acquisition of residual lower limb}

The first and most important phase is the $3 \mathrm{D}$ acquisition of residual limb made using Magnetic Resonance Imaging (MRI) or a low cost RGB-D depth sensor. In this application it is an essential aspect to have the most faithful model of the real object. MRI gives us the possibility to reconstruct not only the external shape, but also the internal structure with bones and soft tissues [1]. This technique allows getting more information respect to a $3 \mathrm{D}$ scanner acquisition because we can evaluate the behavior of socket also according to the internal structure of the residual limb.

\subsubsection{Socket design}

The prosthetist is guided step-by-step by the system that applies in automatic or semi-automatic way the rules and the modelling procedures. Actually, it embeds a set of design rules and procedures (e.g., where and how to modify the socket shape) and makes available a set of interactive virtual tools to manipulate the socket shape according to traditional procedures (Figure 3). Furthermore, we have developed a module that exploits hand-tracking devices (e.g., Leap Motion device [2]) in order to emulate the design operation as traditionally made by orthopedic technicians who use their hands to shape the socket. Beyond the technical challenge of creating such a complex ICT tool, the relevance of this part is mainly due to the inner connection it has with the medical application. The module has been built exploiting orthopedic knowledge extracted from physicians' interviews and best cases, coded and embedded into the system. Moreover, the real applicative condition in a prosthetic lab has been kept in high consideration while designing the process and the user's interface. This should ease the transition to a digital way of designing the socket.
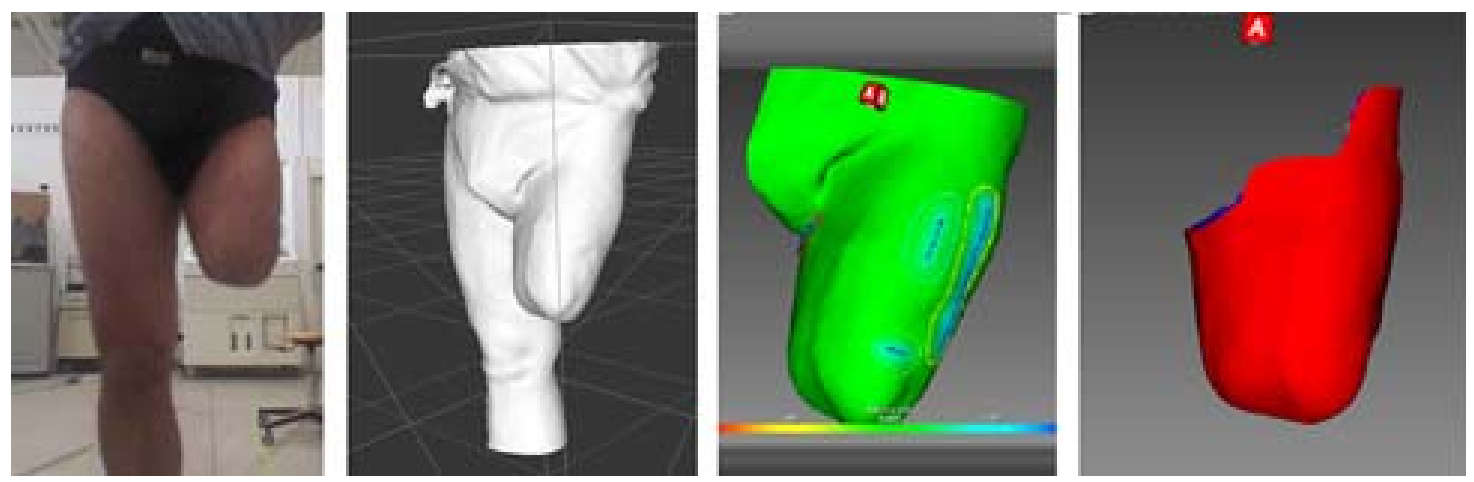

Figure 3 - Socket reconstruction process using MS Kinect and Skanect.

\subsubsection{Socket Test}

The design of the socket is performed taking into account several patient's features and, thus, the result achieved is supposed to be very close to the desired one. By the way, there are some characteristics that can be hardly modeled in the design phase, as the residual limb mechanical properties and its deformation during the donning and the gait. Such results can be achieved by means of a contact pressure numerical simulation. The main challenge of this phase has been to implement an automatic procedure to provide the medical staff with Finite Element simulation results without the intervention of a simulation expert. Moreover, the outcome of simulation highlights eventual pressure peaks or other issues that can be passed to SMA in order to be automatically fixed. The numerical simulation required the definition of a number of parameters guiding the process and of simulations rules related to geometric model, mesh, material characterization, analysis steps, and boundary conditions. They have been derived from an extensive analysis of the state of the art, from the experience acquired in previous research activities, and by balancing accuracy of results and computational costs. As said, simulation results can be handled in background so that no expert is required, by the way, to control the shape change operated by SMA a graphical representation of the pressure distribution can be shown. Once the iterations among the design and test phases is completed, i.e. when the patient's load does not create critical pressure conditions, the output can be generated in order to proceed with the socket manufacture. SMA automatically exports two STL files, corresponding to the load and off-load zones and, thus, the different material to be used for the manufacturing. 


\section{3D scanning state of art}

Technological progress made possible to introduce the concept of 3D scanning in the industrial world nowadays. Scanning means to create a virtual copy of the document or object considered. If a few years ago it was all about 2D scanning, nowadays it is possible to introduce a more evolved tool that allows three-dimensional scans. 3D scanners arrived and invaded the global market for the reason that they offer great opportunities by allowing users to quickly create a 3D model that can be used in any $C A D$ software. The evolution of $3 \mathrm{D}$ scanning systems has determined a large range of commercial solutions with different costs depending on their precision, accuracy, operating principle and other features. In the following paragraphs are illustrated possible classifications which explain the differences between mobile scanner technologies.

\subsection{Operating principles}

All scanning technologies are related to only two operating principles [3] based on light projection that are laser and structured light projection. Really, either operating principles use the triangulation method to obtain the distances from the sensor and to create the point cloud of the scanned object. This method consist in the calculus of the distance of a point, knowing the projection and the receiving angle using trigonometric equations.

\subsubsection{Laser scanning technologies}

Laser scanner is formed by a laser emitter and a camera (Figure 4a). These components are displaced with an angle between them that define the field of view. The emitter projects a laser stripe on the object to scan, it is framed by the camera and using triangulation method, it is possible to calculate the distance from each point of the object. The most important benefits of this system are high precision and accuracy in comparison between real object and scanned model. This operating principle have also two disadvantages that are the high cost of the hardware components and the scan duration that is high for the small scan area.

\subsubsection{Structured light technologies}

These scanners are based on structured light technology (Figure 4b) that consists in the projection of light pattern of IR/laser beams and using triangulation formulas, they permit to evaluate the depth value for each point of the pattern. These scanners include a pattern projector and a light sensor. This technology offers the possibility to capture very large field of view in a single scan acquisition and to increase the precision comparing data with the previous and following frames. Another type of scanners that use a light pattern is based on flash bulb and a camera. The bulb flashes a light pattern onto the object and the camera records this pattern. The distortion in the light pattern, due to the specific curvature of the object is then translated into a 3D image. This type of light is not dangerous for human eyes like laser beams.
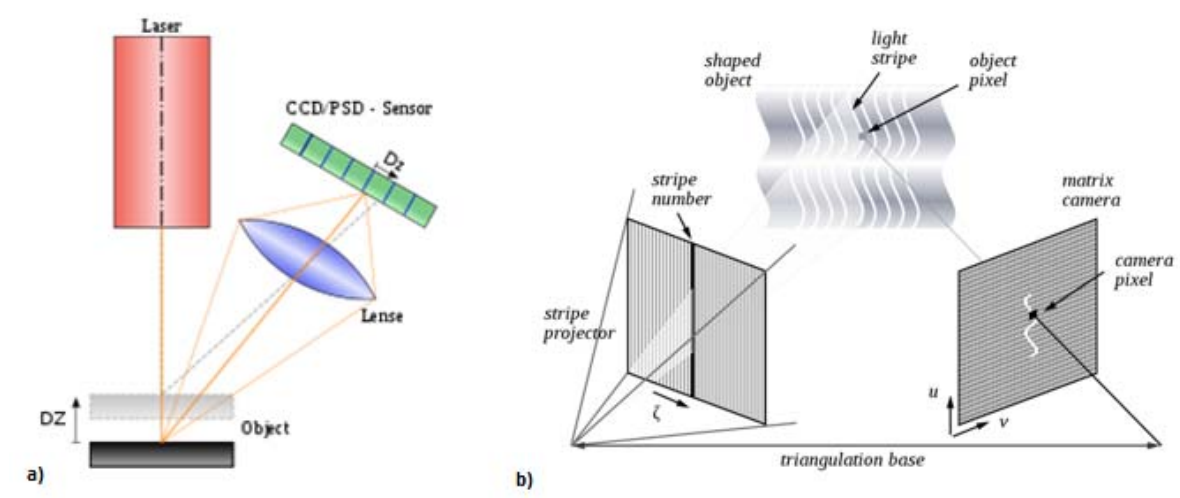

Figure 4 - a) Laser triangulation b) Structured light technology (image courtesy wysiwyg3d.com)

\subsection{Precision and accuracy}

3D scanner can be classified from an application point of view according to precision and accuracy class. Each scanner ensures a level of precision and accuracy depending on operating principle and structure. Accuracy means the trend to converge of the measurement value towards the average value, while precision consists in having all measurements in a very restricted range around the average value. There is a strength relation between the level of this two features and the price of the scanner. In this way it is possible to associate low price to low precision and accuracy and vice versa. 


\subsubsection{Low cost}

The first class includes all low cost scanners. The most of them come from the video-gaming world and for this reason their price is very low: these systems cost less than one thousand euros. Otherwise, the precision of this system is related to the precision required in video games: it depends on environment conditions, light and distance from the object. Anyway, the precision value is around $5-10 \mathrm{~mm}$, that is not comparable to laser scanner, but it has many advantages like illustrated in [4]. In this class, all devices use structured light projection as operating principles with IR beams. The most representative scanner of this section is the MS Kinect v1 (Figure 5a) that is the sensor integrated with XBOX 360. It permits to scan objects with a lot of software and to elaborate a triangular mesh model. Its features and its very low cost are suitable for a lot of medical applications such as rehab application, postural control and gait analysis as explained in $[5,6,7]$

\subsubsection{Medium cost}

Medium cost class includes 3D scanners from 1.000 to 15.000 euros. Hardware improvement permits an increase of precision with value from 0.1 to $1 \mathrm{~mm}$. This value is also validate by the accuracy index (around $0.1 \mathrm{~mm}$ ). These scanners are dedicated to artistic and industrial scopes. This group uses as operating principle either structured light technology and laser projection. A good example of this group is the FARO CAM2 Freestyle scanner (Figure 5b).

\subsubsection{High cost}

All devices with a cost over 15.000 euros belong to this group. These devices are intended for industrial scopes and for this reason they assure a high precision in each measurement. In fact, guaranteed precision is about 0.005 to $0.01 \mathrm{~mm}$. Obviously, high performances are directly proportional to the price of the scanner: depending on the application, the price could reach hundreds of thousands euros. For example, CREAFORM HandySCAN 700 (Figure 5c) offers the possibility to scan object with a precision around $0.05 \mathrm{~mm}$ and costs up to fifty thousand euros [8]. All these scanners are based on laser projection system and using triangulation they can calculate precise measurements. Some of them mount also a contact hard probe to measure the object in particular points and to integrate non-contact data with the aim to obtain a more precise 3D model.

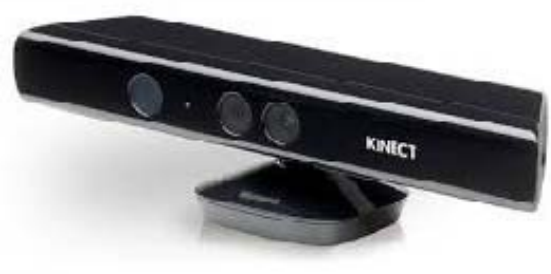

a)

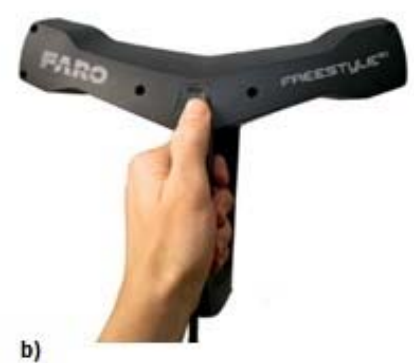

b)

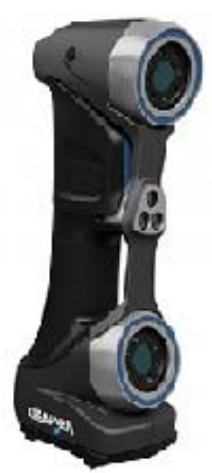

Figure 5 a) MS Kinect v1 b) Faro CAM2 Freestyle c) CREAFORM HandySCAN 700

\section{Application}

MS Kinect v1 sensor allows scanning the residual lower limb of a patient, exporting a STL file. This tool requires the user to takes special cares to optical occlusions and light conditions. In our tests, the subject is in a comfort and natural standing position and he is not wearing the lower limb prosthesis.

The residual limb must be inclined laterally to allow a complete visual to the sensor without obstructions by the other leg. The subject can lean on tripods placed near him (Figure 6). We consider this position because it emulates the natural standing position and the center of mass of the body is the same. Thus, internal forces are not introduced and soft zones are acquired in relaxed position. In very soft zones it is necessary to consider the effect of gravity and this position helps on stability and stillness. Actually, an important aspect for 3D scanning of human body concerns imperceptible movements that are impossible to avoid. Even more a natural and stable posture helps to reduce them and therefore the scan is more precise. 
3D acquisitions with Kinect v1 could be made with commercial software such as Faro Scenect [9], Artec Studio [10] and others, but we prefered Skanect by Occipital [11] for its convenient price-performance ratio. This software, after a preliminary phase of setup of bounding box size, enables to make scan by moving the sensor around the object. In our case, the user has to move the Kinect around patient following, where possible, a circular path. S/He has to pay attention to the zone between legs and eventually around scars. In real time, Skanect software create a virtual model of the limb scanned: through colors (green/red) it provides a direct evaluation of which zones are good scanned and vice versa. In this phase it is also possible to apply filters or reduce numbers of points mapped to simplify mesh geometry.

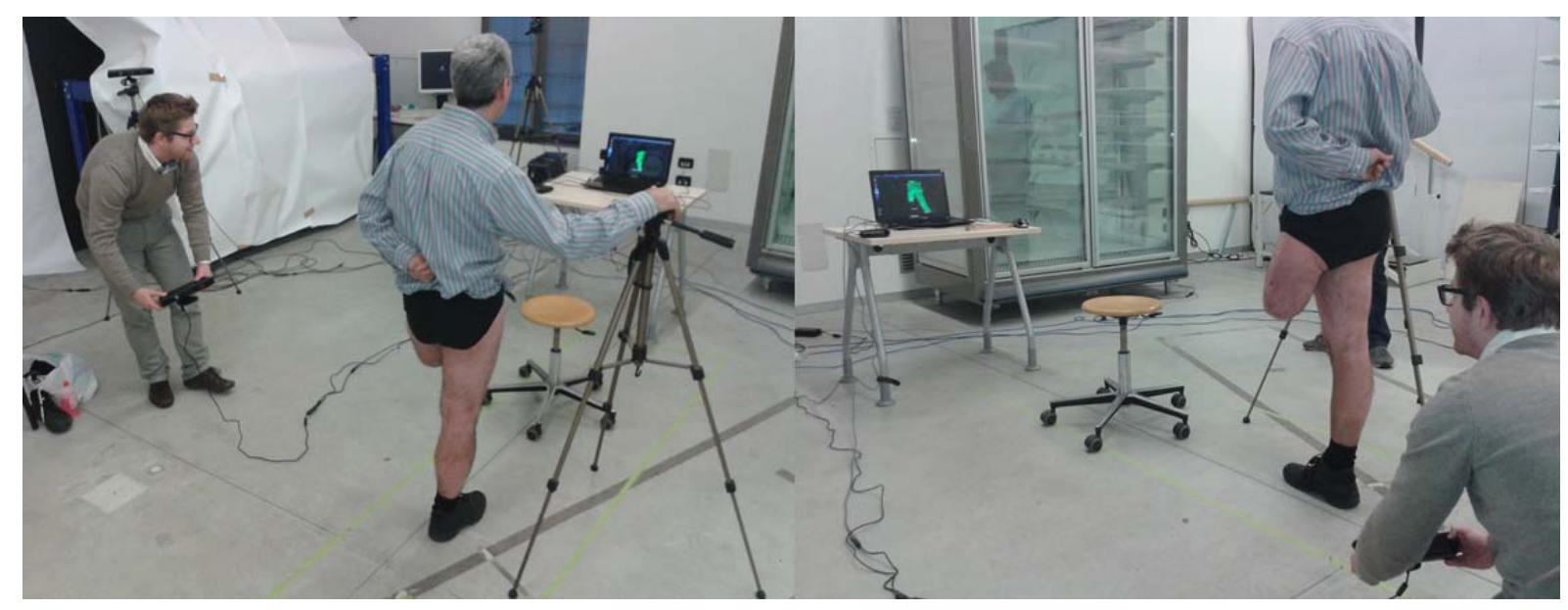

Figure 6 - 3D scanning procedure of a lower limb patient with MS Kinect v1

Anyway, it is important to clean the meshed file removing all double points and faces overlapping that could create some trouble. Using this technology, in addiction to geometry, a skin texture is provided that permits to identify scars due to the amputation. This factor helps to creating off-load zones, including scars position.

The next step is the analysis with SMA tools. Socket Modelling Assistant allows designing lower limb socket starting from the residual limb geometry as explained previously. It also provides a model for FE analysis based on MRI internal structure and basing knowledge on it, we can define load and off-load zones, exported in two STL files.

\section{Discussion}

The presented method could be applied for medical purpose in orthopedic field. Considering lower limb prosthesis, there is no interest to high precision scan of the residual limb. In fact when we do a scan of the residual limb, we are scanning a soft and deformable component and thus, it does not need a perfect acquisition with a precision of a fraction of millimeter, but it is enough to have a precision of a few millimeters. For this reason we chose MS Kinect v1 (Figure 5a) that use IR structured light technology to obtain triangular mesh of the scanned object. This choice involves the disadvantage to have a low precision in the reconstruction of the mesh. This means that the reconstruction algorithm of the model have to connect points in several triangular shapes starting from the acquired point cloud. MS Kinect v1 in relation to Skanect software does not provide a homogenous meshed model with the limitation to round the real shape, due to a not precise discretization. A more high-priced scanner could solve this approximation and create a manifold model based on triangles with same areas. This factor simplifies FE analysis, reducing computational time. Anyway, the mesh provided by Kinect is enough clean and accurate only if you pay attention to critical areas to scan, during acquisition phase (i.e. holes, scars, folds, etc.). (Figure 7) 


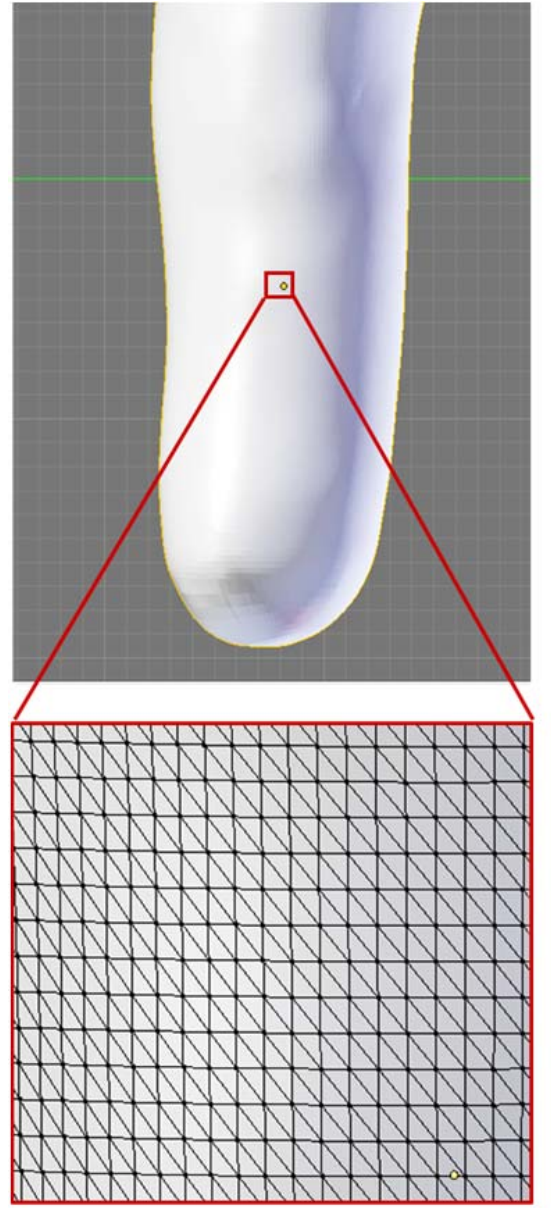

(a)

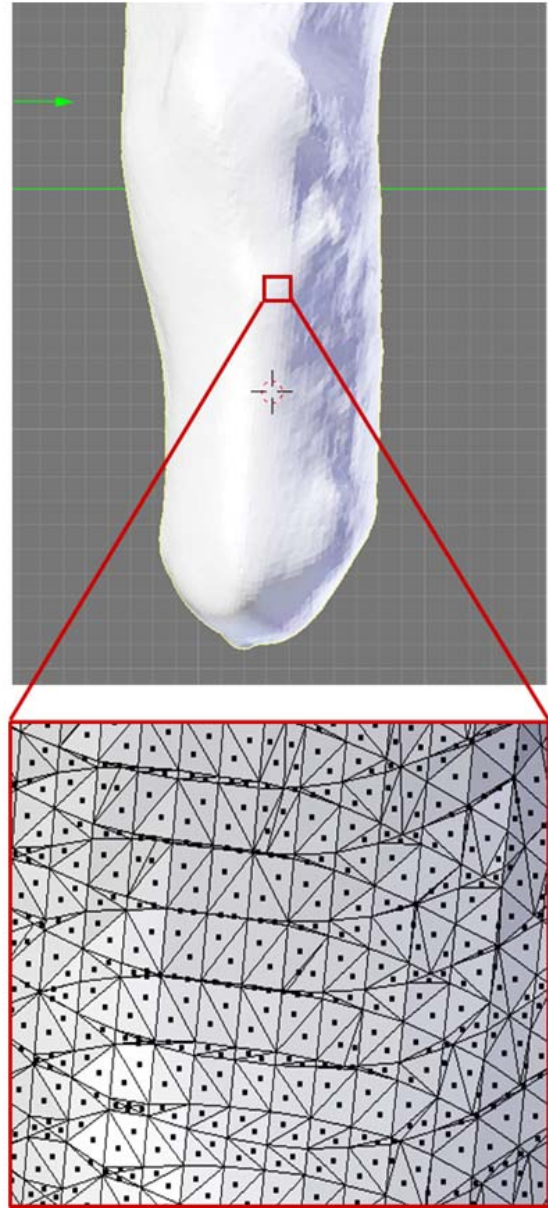

(b)

Figure 7 - Transtibial lower limb residual model taking with a) commercial laser scanner and with b) MS Kinect v1

Kinect optical sensor and each other 3D optical scanner have the feature to scan the limb in a relaxed position which does not create or minimizes deformation. This is not possible with MRI exam: it is performed with the patient in a supine position on the examination table for all its duration. Patient limbs are obviously deformed for the contact with the table, but this is not a problem for the aim of the exam because the internal structure is not modified. MRI external shape of residual limb suffers of a flattening. (Figure 8)

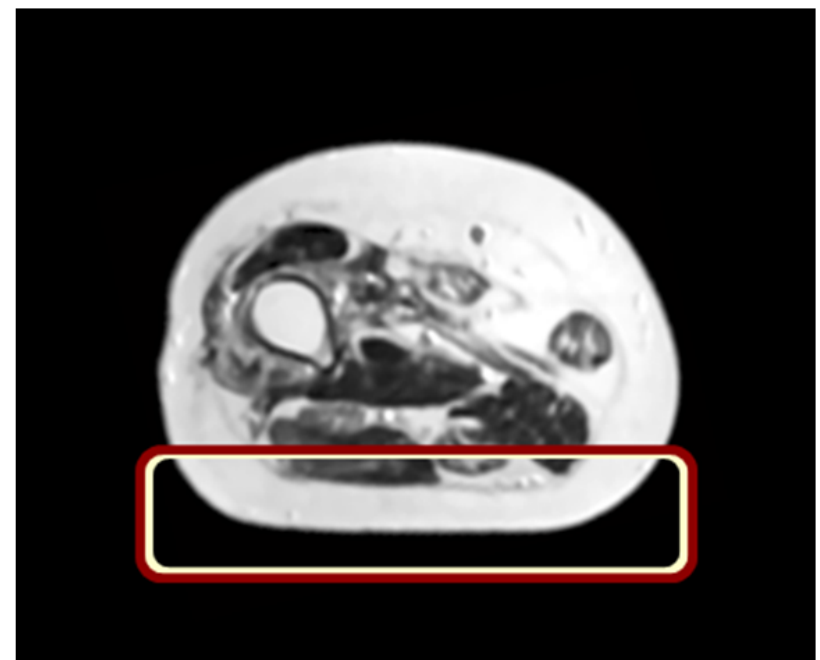

Figure 8 - MRI leg cross section: flattening of soft tissues 
Kinect could be also used to evaluate differences between virtual 3D model and real socket. Executing a new scan at the end of the production process it is possible to check the dimension correspondence between the designed and the realized socket. Scanning a socket is not an easy operation because it need to consider that Kinect captures from a distance from camera lenses of about $50 \mathrm{~cm}$. This limitation requires that user keeps a certain distance from the object and in particular in dark or undercut areas it difficult to scan and create a mesh without any holes. Other problems come from the material of some sockets: they have some areas made of transparent material that creates problems in the scanning phase with Kinect, for this reason it is necessary to temporarily cover them with a dull film.

For example, it may be useful to archive patients' history recording also the shape evolution of the residual limb. In this way, a comparison could be done between old and new socket.

Another useful test is the scan of the 3D printed socket exported from SMA software. This step allows eventually modifying geometry on the 3D printed check socket before realizing the final one. 3D printing is an emerging method that permits a lot of important application and that surely we will be evaluated in future works.

\section{Conclusions}

The variety of $3 \mathrm{D}$ scanning solutions has not yet been exploited in all the potential applications. This paper shows a way in which such technologies can be introduced in the design of custom fit products. The chance for technicians to develop a product starting from the digital shape of the final user is changing the design paradigm. The main benefits consist in shortening the time required to finalize the product and working on a virtual prototype instead of a physical one, thus exploiting the benefits of a digital environment until the final manufacturing.

Scanning technologies are different in precision, accuracy and market price depending on their application target. In the context of lower limb design exposed in the paper there is no need for high performance and, thus, low-cost solutions derived from the video gaming industry are suitable for the design of custom fit components as the socket of the prosthesis.

\section{References}

[1] C. Rizzi, G. Colombo, G. Facoetti, A. Vitali, A. Zanello, "Automatic 3D reconstruction of transfemoral residual limb from MRI images", in $\mathrm{HCl}$ International Conference, Las Vegas, Volume: Lecture Notes in Computer Science (including subseries Lecture Notes in Artificial Intelligence and Lecture Notes in Bioinformatics) 2013, http://dx.doi.org/10.1007/978-3-642-39182-8 38

[2] C. Rizzi, G. Colombo, G. Facoetti, A. Vitali, "Socket Virtual Design Based on Low Cost Hand Tracking and Haptic Devices" in VRCAI '13 Proceedings of the 12th ACM SIGGRAPH International Conference on Virtual-Reality Continuum and Its Applications in Industry, Hong Kong 2013 http://dx.doi.org/10.1145/2534329.2534351

[3] N. D'Apuzzo, "State of the art of the methods for static 3d scanning of partial or full human body", in Homometrica Consulting, Zurich, Switzerland 2006

[4] C. Park; S. Kim; D. Kim; S. Oh, "Comparison of plane extraction performance using laser scanner and Kinect," in Ubiquitous Robots and Ambient Intelligence (URAI), 2011 8th International Conference on , vol., no., pp.153,155, 23-26 Nov. 2011 http://dx.doi.org/10.1109/URAl.2011.6145951

[5] Y. Changa, S. Chenb, J. Huangc, "A Kinect-based system for physical rehabilitation: A pilot study for young adults with motor disabilities", in Research in Developmental Disabilities, Volume 32, Issue 6, November-December 2011, Pages 2566-2570, http://dx.doi.org/10.1016/i.ridd.2011.07.002

[6] R.A. Clarka, Y. Puab, K. Fortina, C. Ritchiea, K. E. Websterc, L. Denehya, A.L. Bryanta, "Validity of the Microsoft Kinect for assessment of postural control", in Gait \& Posture, Volume 36, Issue 3, July 2012, Pages 372-377, http://dx.doi.org/10.1016/i.gaitpost.2012.03.033

[7] D. Regazzoni, C. Rizzi, C. Comotti, F. Massa, "Towards automatic gait assessment by means of rgb-d mocap", in ASME International Design Engineering Technical Conferences \& Computers and Information in Engineering Conference IDETC/CIE 2015, August 2-5, 2015, Boston, Massachusetts, USA [in press]

[8] http://3d-scanners.ireviews.com/

[9] SCENECT by Faro, http://www.faro.com/it-it/scenect/scenect

[10] ARTEC STUDIO by Artec, http://www.artec3d.com/hardware/primesense/

[11] SKANECT 3D Scanning software by Occipital, http://skanect.occipital.com/ 